\title{
To Evaluate the Correlation between Intracranial Pressure and Intraocular Pressure in Neurological Patients: A Prospective Cohort Study

\author{
Pragati Garg ${ }^{*}$, Smriti Diwedi and Ritika Mullick
} \\ Department of Ophthalmology, Era's Lucknow Medical College and Hospital, India
}

*Corresponding author: Dr. Pragati Garg, Professor, HOD, Department of Ophthalmology, Era's Lucknow Medical College and Hospital, Lucknow, India, Tel: 91-941539506

\begin{abstract}
Background: Anatomical proximation of eye with the intracranial space indicates potential relation between cerebrospinal fluid pressure and intraocular pressure.

Aims: Based on their similarity and apparent relationship, non-invasive measurement of intraocular pressure might substitute for lumbar puncture for measuring cerebrospinal fluid pressure in near future.

Settings and design: Hospital based prospective cohort study.

Methods and material: 120 patients after their informed consent and approval by institutional ethical committee. All patients who were above 18 years; could give consent and who underwent lumber puncture for any neurological symptom or diagnostic reasons and intraocular pressure was measured using Schiotz tonometer, were eligible for this study.
\end{abstract}

Statistical analysis used: Chi square test between ordinal (intraocular pressure) and categorical (intracranial pressure) values, and linear regression for ordinal (both intraocular pressure and intracranial pressure) values.

Results: $68.5 \%(89 / 120)$ of patients had normal intracranial pressure between 5 and $15 \mathrm{mmhg}$, while $29.2 \%$ (38/120) of patients had elevated intracranial pressure of more than 15 mmhg. regarding mean intraocular pressure values, $6.9 \%$ $(9 / 120)$ of patients had lower intraocular pressure, less than 10 mmhg: $90.8 \%(118 / 120)$ of patients with normal intraocular pressure between 10 and $21 \mathrm{mmhg}$, the other $2.3 \%(3 / 120)$ of patients with higher intraocular pressure more than $21 \mathrm{mmhg}$. our results showed that intracranial pressure was significantly and positively correlated with intraocular pressure intracranial pressure was correlated significantly with intraocular pressure of the right eyes and intraocular pressure of the left eyes and mean intraocular pressure (MIOP) of both eyes.
Conclusions: The findings in our study are statistically significant suggest that the elevated intracranial pressure may have acted as a counter pressure across the lamina cribrosa to compensate for the elevated intraocular pressure. This correlation is helpful in proving that intraocular pressure can replace invasive modalities for intracranial pressure in the near future.

\section{Keywords}

Non-Invasive, Intraocular Pressure, Intracranial Pressure

\section{Introduction}

Raised intra-cranial pressure is one of the signals of a neurological condition. Lumbar puncture is still the gold standard procedure for measurement of CSF but there are various risks associated with it [1]. This direct invasive method can cause cerebral herniation, intracranial haemorrhage, infection and post procedure headache, while also being difficult to execute properly. Intracranial pressure (ICP) is a product of the volume and compliance of the craniospinal contents [2].

There are non-invasive alternatives for CSF pressure measurement such as trans-cranial doppler (TCD), visual evoked responses (VERs), brain stem auditory responses (BAERs), ophthalmodynamometer, scalp blood flow (SBF) measured by Laser Doppler and impedance audiometry. These methods are still being explored and require further validations [1].

Anatomical proximation of eye with the intracranial space indicates potential relation between cerebrospi- 
Table 1: List of Neurological Causes presented by the Patients.

\begin{tabular}{|l|l|l|l|}
\hline N o & Cause of lumbar puncture & N & Percentage \\
\hline 1. & Headache of Unknown cause & 38 & 31.6 \\
\hline 2. & Meningitis review & 22 & 18.3 \\
\hline 3. & Convulsions & 20 & 16.6 \\
\hline 4. & Facial palsy & 4 & 3.3 \\
\hline 5. & Loss of memory & 2 & 1.6 \\
\hline 6. & Language & 2 & 1.6 \\
\hline 7. & Diplopia & 4 & 3.3 \\
\hline 8. & Encephalitis & 8 & 6.6 \\
\hline 9. & Normal pressure hydrocephalous & 2 & 1.6 \\
\hline 10 & Neurosyphilis & 4 & 3.3 \\
\hline 11 & Neurocysticercos is & 2 & 1.6 \\
\hline 12 & Venus sinus thrombosis review & 8 & 6.6 \\
\hline 13 & Mental disorder & 4 & 3.3 \\
\hline & Total & 120 & \\
\hline & & & \\
\hline
\end{tabular}

nal fluid (CSF) pressure and intraocular pressure (IOP) [3]. Also, the chemical composition of aqueous humour and the CSF are strikingly similar. Both are clear fluids with low portion content and play similar roles, to protect and provide nourishment to the surrounding structure. It has been found that the average IOP and CSF pressure are quite similar: the average IOP being about $15 \mathrm{mmHg}$ and the average CSF pressure about $11 \mathrm{mmHg}$ [4]. Based on their similarity and apparent relationship, non-invasive measurement of IOP might substitute for lumbar puncture for measuring CSF pressure in near future. Some recent studies suggest that non-invasive measurement of IOP for indirect estimation of CSF pressure is highly acceptable and could be used as screening tool for measuring CSF pressure in neurological patients.

This motivated us to explore findings if a non-invasive method can be a good substitute for the existing invasive methods of measuring CSF pressure. We studied 120 patients from our Emergency Department with various neurological symptoms. Then IOP was measured with the help of Schiotz tonometer and CSF pressure with LP to look for the possible correlation between the two.

\section{Material and Methods}

This is a hospital based prospective cohort study conducted in a tertiary care hospital from July 2014 to January 2016 on 120 patients after their informed consent and approval by institutional ethical committee.

All patients who were above 18 years: could give consent and who underwent LP for any neurological symptom or diagnostic reasons, were eligible for this study.

Exclusion criteria included patients with history of glaucoma, intraocular surgery or intracranial surgery, spinal cord disease, or ocular disease that can influence IOP and patients on medication such as mannitol, beta blockers, carbonic anhydrase inhibitors which directly or indirectly can influence the IOP and ICP.

Total of 120 patients after detailed proper history and examination were included on who lumbar punc-
Table 2: Patients' Data.

\begin{tabular}{|l|l|l|l|}
\hline & $\begin{array}{l}\text { Min } \\
\text { N }\end{array}$ & Max & $\begin{array}{l}\text { MEAN+ } \\
\text { st (dev) }\end{array}$ \\
\hline Age (year) & 19 & 63 & $42.4 \pm 12.02$ \\
\hline $\begin{array}{c}\text { IOP } \\
\text { OD }(\mathrm{mmHg})\end{array}$ & 8.5 & 28.5 & $1919.33 \pm 3.61$ \\
\hline $\begin{array}{l}\text { IOP } \\
\text { OS }(\mathrm{mmHg})\end{array}$ & 8.5 & 24 & \\
\hline $\begin{array}{l}\text { Mean } \\
\text { IOP }(\mathrm{mmHg})\end{array}$ & 8.5 & 26.4 & $1919.31 \pm 3.42$ \\
\hline
\end{tabular}

ture was done because of various neurological causes (Table 1).

Before lumbar puncture IOP was measured using the schiotz tonometer under local anaesthesia in both the eyes and mean IOP was calculated. Before recording the IOP, the tonometer was cleaned and then placed on the test block (testing procedure) to look for any error. The patient was sedated of necessary for proper measurement and patient safety (to stop intracranial and hemodynamic response). Midazolam was used for sedation in a dose of $2-5 \mathrm{mg}$ intravenously. After anaesthetizing the cornea with xylocaine $4 \%$, reliable pressure values were recorded when the pointer had shown a pulse. According to the manufacturer, the pressure of a healthy eye was approximately $16 \mathrm{mmHg}$. The cut-off point for normal IOP was defined as $21 \mathrm{mmHg}$.

A standard LP technique was used in all patients. Each patient was placed in the lateral decubitus position and either $L 3$ to $L 4$ to $L 5$ interspace was identified and anaesthetized. A 3.5 inch, 20-gauge spinal needle with a three-way stopcock was inserted into the subarachnoid space. A $550 \mathrm{~mm}$ manometer was attached to the stop clock and the column of CSF was allowed to equilibrate. The patient was asked to remain still and not to speak. The meniscus of the CSF was read and reported in millimetres of water. The value was converted to $\mathrm{mm}$ of mercury to allow comparison with IOP and for data analysis $\left(1 \mathrm{mmHg}=13.6 \mathrm{~mm} \mathrm{H}_{2} \mathrm{O}\right)$.

Simultaneous measurement values of IOP and ICP were documented in protocol. To determine the correlation between CSF pressure and IOP we calculated the linear correlation coefficient and the spearman rank order correlation coefficient. The idea of using two statistical methods in this study was to augment the reliability of results (injury).

Table 2 shows the data of 120 subjects enrolled for the study, where the mean age was 42 years with standard deviation of 12 years. The minimum IOP was $8.5 \mathrm{mmHg}$ in both the right and the left eyes. The maximum IOP recorded in the right eye was $28.5 \mathrm{mmHg}$ and in the left eye was $24.4 \mathrm{mmHg}$. The Mean IOP in the right eye was $19 \mathrm{mmHg}$ and in the left eye was 19 $\mathrm{mmHg}$. 

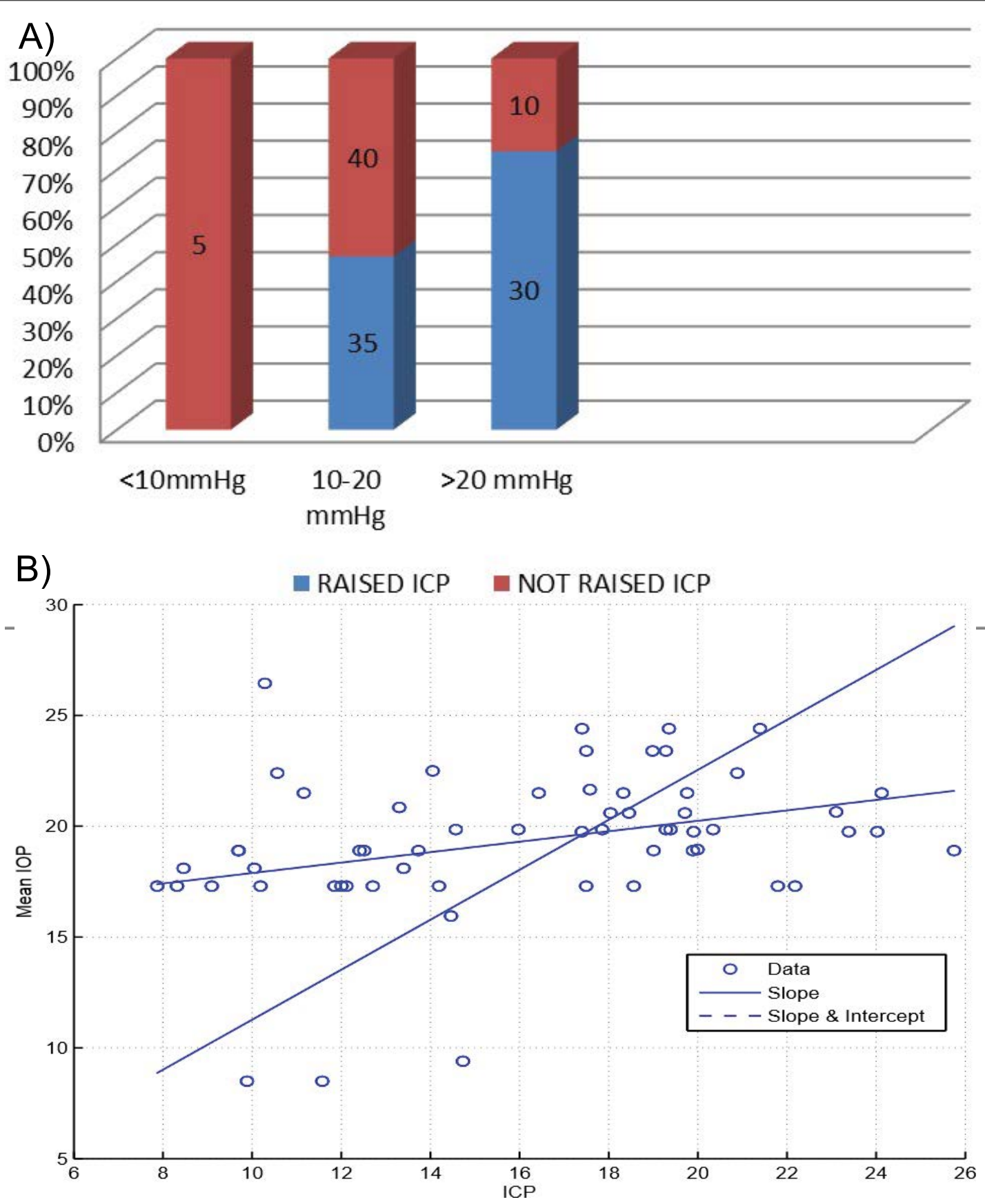

Figure 1: ICP vs. Mean IOP. A) Chi square test; B) Linear Regression.

\section{Results}

The study was conducted on 120 patients, out of which 68 (53\%) were males, and 52 (43\%) were females. In all of them LP was done, and it was found that $68.5 \%$ (89/120) of patients had normal ICP between 5 and 15 $\mathrm{mmHg}$, while $29.2 \%$ (38/120) of patients had elevated ICP of more than $15 \mathrm{mmHg}$. Regarding MIOP values, $6.9 \%(9 / 120)$ of patients had lower IOP, less than 10 mmHg: $90.8 \%(118 / 120)$ of patients with normal IOP between 10 and $21 \mathrm{mmHg}$, the other 2.3\% (3/120) of patients with higher IOP more than $21 \mathrm{mmHg}$.

We ran two sets of analysis: Chi square test between ordinal (IOP) and categorical (ICP) values, and linear regression for ordinal (both IOP and ICP) values (Figure 1).

Our results showed that ICP was significantly and positively correlated with IOP ICP was correlated significantly with IOP of the right eyes and IOP of the left eyes and mean IOP (MIOP) of both eyes. But no correlation between ICP and age were found.

We analysed data for ICP and mean IOP with linear regression from the data, the coefficient of determination $\mathrm{R}^{2}$ was found to be 0.876 which shows a high correlation between ICP and mean IOP. Where $y$ represents the calculated values and $y$ is the mean $R^{2}$ lies between $[0,1]$ and a higher value denotes a higher correlation.

\section{Discussion}

Intracranial pressure measurement via lumbar puncture is still the gold standard for diagnosing neurological and neuro-ophthalmic conditions. 
Various non-invasive methods are proposed namely $\mathrm{CT}, \mathrm{MRI}$, Transcranial Doppler sonography, scanning laser tomography of $\mathrm{ONH}$ and venous ophthalmodynamic meter to replace the invasive LP for ICP measurement, but there are limitations with the techniques and therefore none can be considered as a substitute for LP.

In our population-based study we have found that there exists a substantial relation between ICP and mean IOP. ICP was measured by LP and IOP by schiotz tonometer which is positively related to previous studies.

The anatomical explanation for our result is the presence of communication between the intracranial cavity and orbital cavity through the optic canal. While, the physiological basis for our result was that the pressure in the orbital subarachnoid space is correlated with ICP and subarachnoid space can be distended depending on its pressure because of principal of elasticity according to Poisson effect.

The findings in our study is statistically significant suggest that the elevated ICP may have acted as a counter pressure across the lamina cribrosa to compensate for the elevated IOP.

Salman, et al. [5] in his study asserts that IOP measurement with tonometry is a good indicator of raised ICP value. A probable mechanism as per him could be the rise of the ophthalmic venous pressure as a direct effect of the raised ICP. He also concluded that the CSF surrounds optic nerve sheath to the point where the optic nerve enters the orbit so elevation of ICP could be directly transmitted to the eyeball.

Frischng, et al. and Han Y, et al. [6] also found no relation between IOP and ICP.

Kirk T, et al. [7] also did not find any significant relationship between ICP and IOP, in their study the non-invasive IOP measurements did not predict ICP.

Hayreh [8] professes that here is no relation between ICP and IOP and clarifies that there is no anatomical and pathophysiological basis to explain this relationship in his study on rhesus monkey.

Sheeren, et al. found positive relation between the two in patients with various intracranial pathologies but he alleged that the indirect estimation by substituting with IOP measurement is not useful in clinical practice.
Zhen $\mathrm{li}$, et al. [1] found ICP to be significantly correlated to IOP in patients with neurological conditions and with BMI.

Whereas in an advanced study using complicated measures to determine the relationship between invasive continuous monitoring of ICP using intraparenchymal and IOP measurement by schiotz tonometer only 2 patient showed positive relation.

As we are aware of research data where opinions are still divided regarding correlation between ICP and IOP but based on our study we can say there is a lot of promise in pursuing the direction to establish that IOP can be a good replacement for LP for measuring ICP.

\section{Limitations}

However potential limitations of our study need to be mentioned such as schiotz tonometer, tonopen are also subject to human error, IOP also fluctuates diurnally and we have taken the reading prior to LP irrespective of which time of the day.

IOP also varies depending on corneal thickness, axial length, depth of AC which was not possible to measure as general condition of the patient did not permit us to perform them.

\section{References}

1. Li Z, Yang Y, Lu Y, Liu D, Xu E, et al. (2012) Intraocular pressure vs intracranial pressure in disease conditions: $A$ prospective cohort study (Beijing iCOP study). BMC Neurol 12: 66.

2. Ross N, Eynon CA (2005) Intracranial pressure monitoring. Curr Anaesth Crit Care 16: 255-261.

3. Czarnik T, Gawda R, Kolodziej W, Latka D, Sznajd-Weron K, et al. (2009) Associations between intracranial pressure, intraocular pressure and mean arterial pressure in patients with traumatic and non-traumatic brain injuries. Injury 40: 33-39.

4. Berdahl JP (2013) Systemic parameters associated with cerebrospinal fluid pressure. J Glaucoma 22: S17-S18.

5. Salman MS (1997) Can intracranial pressure be measured non-invasively? Lancet 350: 1367

6. Han Y, McCulley TJ, Horton JC (2008) No correlation between intraocular pressure and intracranial pressure. Ann Neurol 64: 221-224.

7. Kirk T, Jones K, Miller S, Corbett J (2011) Measurement of intraocular and intracranial pressure: is there a relationship? Ann Neurol 70: 323-326.

8. Hayreh SS (2016) Pathogenesis of optic disc edema in raised intracranial pressure. Prog Retin Eye Res 50: 108-144. 